\title{
EWMA Chart and Measurement Error
}

\author{
PETROS E. MARAVELAKIS, JOHN PANARETOS AND \\ STELIOS PSARAKIS \\ Department of Statistics, Athens University of Economics and Business, Athens, Greece
}

\begin{abstract}
Measurement error is a usually met distortion factor in real-world applications that influences the outcome of a process. In this paper, we examine the effect of measurement error on the ability of the EWMA control chart to detect out-of-control situations. The model used is the one involving linear covariates. We investigate the ability of the EWMA chart in the case of a shift in mean. The effect of taking multiple measurements on each sampled unit and the case of linearly increasing variance are also examined. We prove that, in the case of measurement error, the performance of the chart regarding the mean is significantly affected.
\end{abstract}

KeY WoRds: Exponentially weighted moving average control chart, average run length, average time to signal, measurement error, Markov chain, statistical process control

\section{Introduction}

Control charts are a well-known tool in today's industry, and Shewhart control charts are the best known of these. Despite their popularity, they are unable to detect small shifts in a process quickly enough. For this reason other charts have been implemented, such as the Cumulative Sum (CUSUM) and the Exponentially Weighted Moving Average (EWMA) charts.

A problem faced in the context of control charts generally is the measurement error variability. This problem is the result of the inability to measure accurately the variable of interest $X$. The use of imprecise measurement devices affects the ability of the control chart to detect an out-of-control situation. Moreover, the variable under interest may be related through a covariate with the measurement system used.

Mittag \& Stemann (1998) examined the effect of measurement error on the joined $\bar{X}-S$ control chart assuming the model of the form $Y=X+\varepsilon$, where $X$ is the actual value of the variable and $Y$ is the measured value because of the random error $\varepsilon$. Linna \& Woodall (2001) extended the preceding model by assuming one with covariates and they investigated the effect of this model on the $\bar{X}$ and $S^{2}$ control charts. Linna et al. (2001) examined the effect of the model with covariates in the case of a multivariate Shewhart chart for the mean.

This paper deals with the performance of the EWMA control chart for the mean under the effect of measurement error, assuming a model with covariates. In the next section, the EWMA chart for the mean is presented. The EWMA

Correspondence Address: Petros E. Maravelakis, Department of Statistics, Athens University of Economics and Business, Athens, 10434, Greece. Email: jgan@aueb.gr 
chart, when we have a model with covariates, is given in the third section 3 . The subsequent two sections introduce multiple measurements and linearly increasing variance in the EWMA chart, when we have the model with covariates, respectively. The methods for evaluating a control charts' performance and the results of the measurement error model in the EWMA are given in the sixth section. Finally, in the appendix, we provide the theory for the computation of the run length distribution and its first moment in our cases.

\section{The EWMA Control Charts for Monitoring the Process Mean}

Let the mean $\mu$ and standard deviation $\sigma$ of a process be known. The EWMA chart for individual observations is defined as

$$
Z_{t}=\lambda \bar{x}_{i}+(1-\lambda) Z_{t-1}, Z_{0}=\mu,
$$

where $\bar{x}_{i}$ is the mean of the sample of observations in time $i=1,2, \ldots$, and $\lambda$ is a smoothing parameter that takes values between 0 and 1 , and $Z_{0}$ is the initial value. When the value of $\lambda$ is close to 0 , the EWMA chart can detect small to moderate shifts in the process mean, when $\lambda$ is close to unity the EWMA can detect large shifts in the process mean and when $\lambda=1$ it is actually the $\bar{X}$ chart. As a starting value, instead of the in-control process mean, we can use the target value. The control limits of this chart are

$$
\begin{aligned}
& \mathrm{UCL}=\mu+L \frac{\sigma}{\sqrt{n}} \sqrt{\left(\frac{\lambda}{2-\lambda}\right)\left[1-(1-\lambda)^{2 i}\right]} \\
& \mathrm{LCL}=\mu-L \frac{\sigma}{\sqrt{n}} \sqrt{\left(\frac{\lambda}{2-\lambda}\right)\left[1-(1-\lambda)^{2 i}\right]}
\end{aligned}
$$

where $L$ is a constant used to specify the width of the control limits, $\mu$ is the mean and

$$
\frac{\sigma}{\sqrt{n}} \sqrt{\left(\frac{\lambda}{2-\lambda}\right)\left[1-(1-\lambda)^{2 i}\right]}
$$

is the standard deviation of $Z_{t}$ when the process is in control. In case the EWMA chart is used for some time, or for simplification, instead of control limits (1) and (2), we may use their limiting values

$$
\begin{aligned}
& \mathrm{UCL}=\mu+L \frac{\sigma}{\sqrt{n}} \sqrt{\left(\frac{\lambda}{2-\lambda}\right)} \\
& \mathrm{LCL}=\mu-L \frac{\sigma}{\sqrt{n}} \sqrt{\left(\frac{\lambda}{2-\lambda}\right)},
\end{aligned}
$$


(see for example Lucas \& Saccucci, 1990). In this case, is the asymptotic standard deviation of $Z_{t}$.

The EWMA chart has attracted the attention of several researchers the last years. Some of the references for an interested reader on this subject are Crowder (1987), Ng \& Case (1989), Champ \& Rigdon (1991), Reynolds (1996), Gan (1998), Steiner (1998, 1999), Borror et al. (1999), Henderson (2001) and Stoumbos \& Sullivan (2002).

\section{The EWMA Chart Using Covariates}

Assume that we have again a process were the true value of the characteristic $X$ under investigation is normally distributed with mean $\mu$ and variance $\sigma^{2}$ when the process is in control. However, we are not able to observe this true value but rather a value $Y$, which is related to $X$ with the formula $Y=A+B X+\varepsilon$, where $A$ and $B$ are constants and $\varepsilon$ is the random error distributed independently of $X$ as a normal random variable with mean zero and variance $\sigma_{m}^{2}$. We assume here that all model parameters are known.

From the formula relating $Y$ and $X$ it is straightforward that $Y$ is normally distributed with mean $A+B \mu$ and variance $B^{2} \sigma^{2}+\sigma_{m}^{2}$. We need to construct an EWMA chart for the measured quantity $Y$ since, in this way, we can keep variable $X$ under control. Assume that at each sampling point we collect $n$ values of $Y$, we calculate the mean of these observations $\bar{Y}_{i}$ and we compute the EWMA statistic $Z_{t}$ using the formula

$$
Z_{t}=\lambda \bar{Y}_{i}+(1-\lambda) Z_{t-1}, Z_{0}=A+B \mu
$$

where $\bar{Y}_{i}$ is the mean of the observations collected at time $i=1,2, \ldots$ and $\lambda$ is again the smoothing parameter.

The control limits are

$$
\begin{aligned}
& \mathrm{UCL}=A+B \mu+L \sqrt{\left(\frac{\lambda}{2-\lambda}\right)\left[1-(1-\lambda)^{2 i}\right] \frac{B^{2} \sigma^{2}+\sigma_{m}^{2}}{n}} \\
& \mathrm{LCL}=A+B \mu-L \sqrt{\left(\frac{\lambda}{2-\lambda}\right)\left[1-(1-\lambda)^{2 i}\right] \frac{B^{2} \sigma^{2}+\sigma_{m}^{2}}{n}},
\end{aligned}
$$

where $L$ is a constant used to specify the width of the control limits and $A+B \mu$ and

$$
\sqrt{\left(\frac{\lambda}{2-\lambda}\right)\left[1-(1-\lambda)^{2 i}\right] \frac{B^{2} \sigma^{2}+\sigma_{m}^{2}}{n}}
$$

are the mean and standard deviation of $Z_{t}$ respectively, when the process is in control. In case the EWMA chart is used for some time, instead of the control limits (5) and (6), we may use their limiting values 


$$
\begin{aligned}
& \mathrm{UCL}=A+B \mu+L \sqrt{\left(\frac{\lambda}{2-\lambda}\right) \frac{B^{2} \sigma^{2}+\sigma_{m}^{2}}{n}} \\
& \mathrm{LCL}=A+B \mu-L \sqrt{\left(\frac{\lambda}{2-\lambda}\right) \frac{B^{2} \sigma^{2}+\sigma_{m}^{2}}{n}},
\end{aligned}
$$

(see for example Lucas \& Saccucci, 1990). In this case,

$$
\sqrt{\left(\frac{\lambda}{2-\lambda}\right) \frac{B^{2} \sigma^{2}+\sigma_{m}^{2}}{n}}
$$

is the asymptotic standard deviation of $Z_{t}$.

\section{Multiple Measurements}

A technique suggested by Linna \& Woodall (2001) in order to decrease the measurement error effect is to take more than one measurement in each sampled unit. Taking several measurements and averaging them leads to a more precise measurement. Moreover, the variance of the measurement error component in the average of the multiple observations becomes smaller as the number of multiple measurements increases. Therefore, ideally, if the number of multiple measurements becomes infinite the variance of the measurement error component will become zero. Consequently, the larger the number of multiple measurements the better, always keeping in mind the additional cost and time needed for these observations. We must also understand that, in the absence of measurement error, multiple measurements will not contribute anything to the control charting methodology, in fact they will add the cost of measuring the extra observations.

In the case of enough multiple measurements, we can assume that our process actually operates without measurement error. However, the cost of extra measurements and time are factors that cannot be overlooked. Therefore, a careful examination of these factors in the specific application we are working on is essential. We have to stress though that the measurement error variance has to be large enough and the two factors small enough for the extra observations to have a practical value.

In order to compute the EWMA statistic we assume that, at each sampling point, we collect $k$ measurements for each of $n$ observations of $Y$, we calculate the overall mean of these observations $\overline{\bar{Y}}_{i}$, and we compute the EWMA statistic $Q_{i}$ using the formula

$$
Q_{t}=\lambda \overline{\bar{Y}}_{i}+(1-\lambda) Q_{t-1}, Q_{0}=A+B \mu,
$$

where $\overline{\bar{Y}}_{i}$ is the mean of the observations collected at time $i=1,2, \ldots$, and $\lambda$ is a smoothing parameter that takes values between 0 and 1 , while $Q_{0}$ is the initial value. Additionally, we assume that the $k$ observations collected at the same sampling unit are independent. If $k=1$, we face the measurement error case discussed in the third section. 
It is straightforward to prove (Linna \& Woodall, 2001) that the variance of the overall mean is

$$
\frac{B^{2} \sigma^{2}}{n}+\frac{\sigma_{m}^{2}}{n k}
$$

Therefore, the control limits are

$$
\begin{aligned}
& \mathrm{UCL}_{\mathrm{Q}}=A+B \mu+L \sqrt{\left(\frac{\lambda}{2-\lambda}\right)\left[1-(1-\lambda)^{2 i}\right]\left(\frac{B^{2} \sigma^{2}}{n}+\frac{\sigma_{m}^{2}}{n k}\right)} \\
& \mathrm{LCL}_{\mathrm{Q}}=A+B \mu-L \sqrt{\left(\frac{\lambda}{2-\lambda}\right)\left[1-(1-\lambda)^{2 i}\right]\left(\frac{B^{2} \sigma^{2}}{n}+\frac{\sigma_{m}^{2}}{n k}\right)},
\end{aligned}
$$

where $L$ is a constant used to specify the width of the control limits and $A+B \mu$ and

$$
\sqrt{\left(\frac{\lambda}{2-\lambda}\right)\left[1-(1-\lambda)^{2 i}\right]\left(\frac{B^{2} \sigma^{2}}{n}+\frac{\sigma_{m}^{2}}{n k}\right)}
$$

are the mean and standard deviation of $Q_{i}$ respectively, when the process is in control. In case where the EWMA chart is used for some time, instead of control limits (9) and (10), we may use their limiting values

$$
\begin{aligned}
& \mathrm{UCL}_{\mathrm{Q}}=A+B \mu+L \sqrt{\left(\frac{\lambda}{2-\lambda}\right)\left(\frac{B^{2} \sigma^{2}}{n}+\frac{\sigma_{m}^{2}}{n k}\right)} \\
& \mathrm{LCL}_{\mathrm{Q}}=A+B \mu-L \sqrt{\left(\frac{\lambda}{2-\lambda}\right)\left(\frac{B^{2} \sigma^{2}}{n}+\frac{\sigma_{m}^{2}}{n k}\right)}
\end{aligned}
$$

\section{Linearly Increasing Variance}

Although the model with covariates considered in the third section 3 assumes constant variance it is not unlikely to have a model with variance that depends on the mean level of the process. Specifically, both Montgomery \& Runger (1994) and Linna \& Woodall (2001) refer to practical problems indicating situations where this phenomenon occurs in industry.

We assume that the variance changes linearly with variable $X$. The model we use is again $Y=A+B X+\varepsilon$ with the same assumptions as in the third section, except that $\varepsilon$ is distributed as a normal variable with mean 0 and variance $C+D \mu$. As in the third section, all model parameters are assumed known. From the relation between $Y$ and $X$ we deduce that $Y$ is normally distributed with mean $A+B \mu$ and variance $B^{2} \sigma^{2}+C+D \mu$. The EWMA statistic will be exactly the same as in the third section. 
We can prove that the control limits of the EWMA statistic are

$$
\begin{aligned}
& \mathrm{UCL}_{\mathrm{v}}=A+B \mu+L \sqrt{\left(\frac{\lambda}{2-\lambda}\right)\left[1-(1-\lambda)^{2 i}\right]\left(\frac{B^{2} \sigma^{2}+C+D \mu}{n}\right)} \\
& \mathrm{LCL}_{\mathrm{v}}=A+B \mu-L \sqrt{\left(\frac{\lambda}{2-\lambda}\right)\left[1-(1-\lambda)^{2 i]}\right]\left(\frac{B^{2} \sigma^{2}+C+D \mu}{n}\right)},
\end{aligned}
$$

where $L$ is again a constant used to specify the width of the control limits and $A+B \mu$ and

$$
\sqrt{\left(\frac{\lambda}{2-\lambda}\right)\left[1-(1-\lambda)^{2 i}\right]\left(\frac{B^{2} \sigma^{2}+C+D \mu}{n}\right)}
$$

are the mean and standard deviation of the EWMA statistic respectively, when the process is in control. When the EWMA chart is used for a suitable number of points in time, instead of the control limits (13) and (14), we can use their limiting values

$$
\begin{aligned}
& \mathrm{UCL}_{\mathrm{v}}=A+B \mu+L \sqrt{\left(\frac{\lambda}{2-\lambda}\right)\left(\frac{B^{2} \sigma^{2}+C+D \mu}{n}\right)} \\
& \mathrm{LCL}_{\mathrm{v}}=A+B \mu-L \sqrt{\left(\frac{\lambda}{2-\lambda}\right)\left(\frac{B^{2} \sigma^{2}+C+D \mu}{n}\right)}
\end{aligned}
$$

\section{Effect of the Measurement Error}

In a control chart we have two objectives. First, when we are in control, we want our chart to signal (false alarm) as we have planned it to do. In statistical terms, we want the chart to operate with the planned probability of the mean plotting outside the control limits if we are in control. Secondly, when the control chart is out of control, we want it to signal as soon as possible. In statistical terms we want the probability of the mean plotting in control if we are out of control to be as small as possible. Different measures for evaluating the performance of a chart concerning the previous two objectives have been proposed. The best known measure is the average run length (ARL), which is based on the run length (RL) distribution. The number of observations (individual data), or samples (data in subgroups), needed for a control chart to signal is a run length or alternatively one observation of the RL distribution. The mean of the RL distribution is the ARL, which is actually the average number of observations needed for a control chart to signal. Alternatively, the ARL is expressed as the average number of observations to signal (ANOS). A measure similar to the ARL is the average time to signal (ATS), which is the average time needed for a 
Table 1. ARL for the covariate model for different values of $\sigma_{m}^{2} / \sigma^{2}$

\begin{tabular}{lrrrrrr}
\hline Shift & No Error & \multicolumn{1}{c}{0.1} & \multicolumn{1}{c}{0.2} & \multicolumn{1}{c}{0.3} & \multicolumn{1}{c}{0.5} & \multicolumn{1}{c}{1} \\
\hline 0 & 370.22 & 370.27 & 370.27 & 370.27 & 370.27 & 370.26 \\
0.5 & 41.13 & 45.22 & 49.26 & 53.23 & 60.96 & 79.06 \\
1 & 10.25 & 11.21 & 12.18 & 13.16 & 15.15 & 20.26 \\
1.5 & 5.18 & 5.57 & 5.96 & 6.36 & 7.16 & 9.20 \\
2 & 3.46 & 3.69 & 3.91 & 4.13 & 4.57 & 5.67 \\
2.5 & 2.65 & 2.80 & 2.94 & 3.09 & 3.37 & 4.08 \\
3 & 2.19 & 2.29 & 2.40 & 2.50 & 2.71 & 3.22 \\
\hline
\end{tabular}

control chart to signal and it is actually a product of the ARL and the sampling interval used in the case of fixed sampling.

In the context of EWMA charts, there are two ways of computing the previously stated measures of performance. The integral equation method and the Markov chain method (see for example Lucas \& Saccucci, 1990; Domangue $\&$ Patch, 1991). The integral equation method is the more accurate one but it cannot be computed in all cases. The Markov chain method can be implemented in those cases where the previous method cannot, but it is not as accurate as the integral equation method unless we discretize the continuity of the process using many steps. In this paper we use the Markov Chain method in all the computations. The theory used for computing the ARL is given in the Appendix.

In Table 1, we can see the ARL results of the covariate model for different values of the ratio $\sigma_{m}^{2} / \sigma^{2}$ where $B=1$. The in control ARL value is the same for all combinations in order to achieve a fair comparison. From the table, we see that there is an increasing effect on the out of control ARL as the ratio of $\sigma_{m}^{2} / \sigma^{2}$ increases. This result is similar to the one in Linna \& Woodall (2001). In Table 2, we can see the ARL results of the covariate model for different values of $B$. The results are displayed with the same parameters as in Table 1 when $\sigma_{m}^{2} / \sigma^{2}=1$. We observe that as the value of $B$ increases, the effect on the ARL diminishes. This result is again in accordance with Linna \& Woodall (2001). Furthermore, in both Tables 1 and 2 the effect of the measurement error on the ARL values lessens as the shift increases. We have to state also that $A$ does not affect the ARL performance in this study.

Table 2. ARL for the covariate model for different values of $B$

\begin{tabular}{lrrrrr}
\hline Shift & No Error & \multicolumn{1}{c}{1} & \multicolumn{1}{c}{2} & \multicolumn{1}{c}{3} \\
\hline 0 & 370.22 & 370.27 & 370.27 & 370.27 & 370.27 \\
0.5 & 41.13 & 79.06 & 51.25 & 45.67 & 42.78 \\
1 & 10.25 & 20.26 & 12.67 & 11.31 & 10.63 \\
1.5 & 5.18 & 9.20 & 6.16 & 5.61 & 5.33 \\
2 & 3.46 & 5.67 & 4.02 & 3.71 & 3.55 \\
2.5 & 2.65 & 4.08 & 3.01 & 2.81 & 2.71 \\
3 & 2.19 & 3.22 & 2.45 & 2.31 & 2.23 \\
\hline
\end{tabular}


Table 3. ARL for multiple measurements $k=5, B=1$ for different values of $\sigma_{m}^{2} / \sigma^{2}$

\begin{tabular}{lrrrrrr}
\hline Shift & No Error & \multicolumn{1}{c}{0.1} & \multicolumn{1}{c}{0.2} & \multicolumn{1}{c}{0.3} & \multicolumn{1}{c}{0.5} & \multicolumn{1}{c}{1} \\
\hline 0 & 370.22 & 370.26 & 370.26 & 370.27 & 370.27 & 370.27 \\
0.5 & 41.13 & 41.96 & 42.78 & 43.59 & 45.22 & 49.26 \\
1 & 10.25 & 10.44 & 10.63 & 10.82 & 11.21 & 12.18 \\
1.5 & 5.18 & 5.25 & 5.33 & 5.41 & 5.57 & 5.96 \\
2 & 3.46 & 3.51 & 3.55 & 3.60 & 3.69 & 3.91 \\
2.5 & 2.65 & 2.68 & 2.71 & 2.74 & 2.80 & 2.94 \\
3 & 2.19 & 2.21 & 2.23 & 2.25 & 2.29 & 2.40 \\
\hline
\end{tabular}

In Table 3, we can see the ARL results for the covariate model with multiple measurements for different values of $\sigma_{m}^{2} / \sigma^{2}$ when $k=5$ and $B=1$. It is obvious that if the practitioner has the ability to take five measurements in each unit then for values of $\sigma_{m}^{2} / \sigma^{2}$ less than 0.3 we may say that the process operates actually without measurement error. For values larger than 0.3 the effect is seriously reduced in comparison to the $k=1$ case, which corresponds to the results in Table 1, even for $\sigma_{m}^{2} / \sigma^{2}=1$. Table 4 presents the results in the case of multiple measurements for different values of $B$. We see that, as the value of $B$ increases, the effect on the ARL diminishes. This result is in accordance with the results in Table 2. Moreover, in Table 5 we have results in the case of multiple measurements for different $k$ values. As the value of $k$ increases, the measurement error effect reduces. However, since the cost and time needed for the extra

Table 4. ARL for multiple measurements $k=5, \sigma_{m}^{2} / \sigma^{2}=1$ for different values of $B$

\begin{tabular}{lrrrrr}
\hline Shift & No Error & \multicolumn{1}{c}{1} & \multicolumn{1}{c}{2} & \multicolumn{1}{c}{5} \\
\hline 0 & 370.22 & 370.27 & 370.26 & 370.28 & 370.27 \\
0.5 & 41.13 & 49.26 & 43.18 & 42.05 & 41.46 \\
1 & 10.25 & 12.18 & 10.73 & 10.46 & 10.33 \\
1.5 & 5.18 & 5.96 & 5.37 & 5.26 & 5.21 \\
2 & 3.46 & 3.91 & 3.57 & 3.51 & 3.48 \\
2.5 & 2.65 & 2.94 & 2.72 & 2.68 & 2.66 \\
3 & 2.19 & 2.40 & 2.24 & 2.21 & 2.20 \\
\hline
\end{tabular}

Table 5. ARL for multiple measurements for different values of $k$

\begin{tabular}{lrrrrr}
\hline Shift & No Error & \multicolumn{1}{c}{5} & \multicolumn{1}{c}{10} & \multicolumn{1}{c}{20} & \multicolumn{1}{c}{50} \\
\hline 0 & 370.22 & 370.27 & 370.27 & 370.26 & 370.26 \\
0.5 & 41.13 & 49.26 & 45.22 & 43.18 & 41.96 \\
1 & 10.25 & 12.18 & 11.21 & 10.73 & 10.44 \\
1.5 & 5.18 & 5.96 & 5.57 & 5.37 & 5.25 \\
2 & 3.46 & 3.91 & 3.69 & 3.57 & 3.51 \\
2.5 & 2.65 & 2.94 & 2.80 & 2.72 & 2.68 \\
3 & 2.19 & 2.40 & 2.29 & 2.24 & 2.21 \\
\hline
\end{tabular}


Table 6. ARL for linearly increasing variance for different values of $D$

\begin{tabular}{lrrrrr}
\hline Shift & No Error & 1 & 2 & 3 & \multicolumn{1}{c}{5} \\
\hline 0 & 370.22 & 370.27 & 370.28 & 370.27 & 370.28 \\
0.5 & 41.13 & 231.40 & 282.70 & 306.34 & 328.76 \\
1 & 10.25 & 102.95 & 161.16 & 198.80 & 244.30 \\
1.5 & 5.18 & 50.14 & 90.14 & 122.03 & 168.52 \\
2 & 3.46 & 28.10 & 53.72 & 76.89 & 115.28 \\
2.5 & 2.65 & 17.79 & 34.49 & 50.85 & 80.43 \\
3 & 2.19 & 12.39 & 23.73 & 35.38 & 57.77 \\
\hline
\end{tabular}

measurements are important factors, the practitioner will have to do a trade-off between these two concerns and the measurement error s/he can put up with. We have to stress here that the results displayed in this case are for the worst case, since we choose $B=1$ and $\sigma_{m}^{2} / \sigma^{2}=1$, which correspond to the most affected combination. Therefore, one may conclude that the results in the other cases will be even better.

The results in the case of linearly increasing variance are displayed on Tables 6 and 7. In Table 6 we have the ARL values when $B=1, C=0$ and $\sigma_{m}^{2} / \sigma^{2}=1$ for different values of $D$. We see that even for small values of $D$ there is a more serious effect than in the no error case. Additionally, as the value of $D$ increases, this effect is getting larger. This result is expected because, as $D$ increases, so does the variance of the error component in the model. In this special case of measurement error, extra precaution is needed because the ability of the EWMA chart to detect fast small shifts is cancelled out. Consequently, serious distortion factors may go undetected for a long time, costing a lot in money, time and credibility. Table 7 presents the ARL results when $B=1, D=1$ and $\sigma_{m}^{2} / \sigma^{2}=1$ for different values of $C$. Analogously to Table 6 , increasing values of $C$ cause an increasing measurement error effect on the ARL. However, this effect is not of the same magnitude as the effect of $D$. This result is also expected since $D$ is multiplied by the mean $\mu$, increasing faster than the error variance as $D$ increases whereas $C$ is just added to this variance.

In all the computations we used 211 states for the Markov Chain method. Moreover, the values of the constants are $\lambda=0.25$ and $L=2.898$. In order to

Table 7. ARL for linearly increasing variance for different values of $C$

\begin{tabular}{lrrrrr}
\hline Shift & No Error & 0 & 1 & \multicolumn{1}{c}{2} & \multicolumn{1}{c}{3} \\
\hline 0 & 370.22 & 370.27 & 370.29 & 370.27 & 370.27 \\
0.5 & 41.13 & 231.40 & 239.08 & 245.96 & 252.14 \\
1 & 10.25 & 102.95 & 110.13 & 116.95 & 123.44 \\
1.5 & 5.18 & 50.14 & 54.53 & 58.84 & 63.06 \\
2 & 3.46 & 28.10 & 30.72 & 33.34 & 35.95 \\
2.5 & 2.65 & 17.79 & 19.43 & 21.09 & 22.75 \\
3 & 2.19 & 12.39 & 13.49 & 14.59 & 15.71 \\
\hline
\end{tabular}


detect small shifts fast the $\lambda$ value usually used is 0.1 or less. However such small values are not able to detect small to moderate shifts and this is the reason for the $\lambda$ value chosen. Note also that in all the cases the control limits used are the ones with the limiting values.

\section{Conclusions}

In this paper, the performance of the EWMA control chart for the mean, when there is a measurement error effect, assuming a model with covariates, was presented. It was found that this error can affect the ARL performance of this chart. Multiple measurements proved to be a solution to this problem. However, the extra money and time needed is another problem. A properly designed economic study on this matter in each specific problem may reveal the possibility of such an action. On the other hand, the additional time needed may not be a problem since, in today's industry, the measurements are usually done in an automated way. Linearly increasing variance was also discussed and proved to be the type of measurement error that affects the performance of the chart to a larger extent.

\section{References}

Borror, C. M., Montgomery, D. C. \& Runger, G. C. (1999) Robustness of the EWMA control chart to non-normality, Journal of Quality Technology, 31, pp. 309-316.

Crowder, S. V. (1987) A simple method for studying run length distributions of exponentially weighted moving average control charts, Technometrics, 29, 401-407.

Champ, C. W. \& Rigdon, S. E. (1991) A comparison of the Markov Chain and the integral equation approaches for evaluating the run length distribution of quality control charts, Communications in Statistics-Simulation and Computation, 20, pp. 191-204.

Domangue, R. \& Patch, S. C. (1991) Some omnibus exponentially weighted moving average statistical process monitoring schemes, Technometrics, 33, pp. 299-313.

Gan, F. F. (1998) Designs of one and two sided exponential EWMA charts, Journal of Quality Technology, 30 , pp. 55-69.

Henderson, R. G. (2001) EWMA and industrial applications to feedback adjustment and control, Journal of Applied Statistics, 28, pp. 399-407.

Linna, K. W. \& Woodall, W. H. (2001) Effect of measurement error on Shewhart control charts, Journal of Quality Technology, 33, pp. 213-222.

Linna, K. W., Woodall, W. H. \& Busby, K. L. (2001) The performance of multivariate control charts in the presence of measurement error, Journal of Quality Technology, 33, pp. 349-355.

Lucas, J. M. \& Saccucci, M. S. (1990) Exponentially weighted moving average control schemes: properties and enhancements, Technometrics, 32, 1-12.

Mittag, H.-J. \& Stemann, D. (1998) Gauge imprecision effect on the performance of the -S control chart, Journal of Applied Statistics, 25, pp. 307-317.

Montgomery, D. C. \& Runger, G. C. (1994) Gauge capability and designed experiments. Part I: basic methods, Quality Engineering, 6, pp. 115-135.

Ng, C. H. \& Case, K. E. (1989) Development and evaluation of control charts using exponentially weighted moving averages, Journal of Quality Technology, 21, pp. 242-250.

Reynolds, M. R. Jr. (1996) Shewhart and EWMA variable sampling interval control charts with sampling at fixed times, Journal of Quality Technology, 28, pp. 199-212.

Steiner, S. H. (1998) Grouped data exponentially weighted moving average control charts, Applied Statistics, 47, pp. 203-216. 
Steiner, S. H. (1999) EWMA control charts with time-varying control limits and fast initial response, Journal of Quality Technology, 31, pp. 75-86.

Stoumbos, Z. G. \& Sullivan, J. H. (2002) Robustness to non-normality of the multivariate EWMA control chart, Journal of Quality Technology, 34, pp. 260-276.

\section{Appendix}

In order to compute the probability density function, the cumulative distribution function and the first moment of the run length distribution of the EWMA chart for the mean we may approximate it as a discrete Markov Chain by dividing the distance between the control limits in $2 m+1$ states, each of which has width $2 \delta$. We say that the statistic $Z_{t}$ remains in state $j$ as long as $S_{j}-\delta<Z_{t}<S_{j}+\delta$ where $-m \leqslant j \leqslant m$ and $S_{j}$ is the midpoint in the $j$ th interval. When $Z_{t}$ crosses the control limits we say that it is in the absorbing state. On the other hand, when the process is in control we say that it is in a transient state.

The transition probability matrix for the EWMA chart for the mean is computed as

$$
\mathbf{P}=\left[\begin{array}{cc}
\mathbf{R} & (\mathbf{I}-\mathbf{R}) \mathbf{1} \\
\mathbf{0}^{\mathrm{T}} & \mathbf{1}
\end{array}\right]
$$

where $\mathbf{R}$ is a sub-matrix containing the transient states, $\mathbf{I}$ is a $t \times t$ identity matrix and $\mathbf{1}$ is a $t \times 1$ vector of unities. The $j k$ th element of the sub-matrix $\mathbf{R}$ is given by $p_{j k}=P\left\lfloor S_{j}-\delta<\lambda y_{i}+(1-\lambda) S_{j} \leqslant S_{j}+\delta\right\rfloor$. In the case of the normal distribution with the assumed model with covariates of our case the probabilities are given by

$$
p_{j k}=\Phi\left[\frac{\left(S_{k j}+\delta\right)-(1-\lambda) S_{\xi}-\lambda(A+B \mu)}{\lambda \sqrt{\left(B^{2} \sigma^{2}+\sigma_{m}^{2}\right) / n}}\right]=\Phi\left[\frac{\left(S_{k j}-\delta\right)-(1-\lambda) S_{\xi}-\lambda(A+B \mu)}{\lambda \sqrt{\left(B^{2} \sigma^{2}+\sigma_{m}^{2}\right) / n}}\right] .
$$

When we have multiple measurements the probabilities are

$$
p_{j k}=\Phi\left[\frac{\left(S_{k j}+\delta\right)-(1-\lambda) S_{\xi}-\lambda(A+B \mu)}{\lambda \sqrt{\left(B^{2} \sigma / n^{2}+\sigma_{m}^{2} / n k\right.}}\right]=\Phi\left[\frac{\left(S_{k j}-\delta\right)-(1-\lambda) S_{\xi}-\lambda(A+B \mu)}{\lambda \sqrt{\left(B^{2} \sigma / n^{2}+\sigma_{m}^{2} / n k\right.}}\right]
$$

and in the case of linearly increasing variance the probabilities are

$$
p_{j k}=\Phi\left[\frac{\left(S_{k j}+\delta\right)-(1-\lambda) S_{\xi}-\lambda(A+B \mu)}{\lambda \sqrt{\left(B^{2} \sigma^{2}+C+D \mu\right) / n}}\right]=\Phi\left[\frac{\left(S_{k j}-\delta\right)-(1-\lambda) S_{\xi}-\lambda(A+B \mu)}{\lambda \sqrt{\left(B^{2} \sigma^{2}+C+D \mu\right) / n}}\right] .
$$

Let $\tau$ denote the run length of the EWMA then $P(\tau \leqslant t)=\left(\mathbf{I}-\mathbf{R}^{t}\right) \mathbf{1}$ and therefore $P(\tau=t)=\left(\mathbf{R}^{t-1}-\mathbf{R}^{t}\right) \mathbf{1}$ for $t \geqslant 1$. The ARL can be computed using the formula $E(\tau)=\Sigma_{i=1}^{\infty} t P(\tau=t)=\left(\mathbf{I}-\mathbf{R}^{-1}\right) \mathbf{1}$. 